\title{
Energy dissipation and self-heating due to microplastic deformation mechanisms at very high cycle fatigue for single-phase ductile metals
}

\author{
Véronique FAVIER ${ }^{1, a}$, Antoine BLANCHE ${ }^{2, b}$, Ngoc Lam PHUNG ${ }^{1, c}$, \\ Nicolas RANC ${ }^{1, d}$, André CHRYSOCHOOS ${ }^{2, e}$
}

\author{
${ }^{1}$ Arts et Métiers Paristech, Laboratoire Procédés et Ingénierie en Mécanique et Matériaux (PIMM), \\ UMR CNRS 8006, 151 boulevard de l'Hôpital 75013 Paris, France \\ ${ }^{2}$ Laboratoire de Mécanique et Génie Civil (LMGC), Université Montpellier 2, CNRS, Place Eugène \\ Bataillon, 34090 Montpellier, France

\begin{abstract}
averonique.favier@ensam.eu, bantoine.blanche@univ-montp2.fr, 'ngoclam250@yahoo.com, dnicolas.ranc@ensam.eu, ${ }^{\mathrm{e}}$ andre.chrysochoos@univ-montp2.fr
\end{abstract}

Keywords: ultrasonic fatigue, copper, persistent slip bands, heating, fatigue limit

\begin{abstract}
This paper aims at a deeper understanding of mechanisms leading to crack initiation in ductile metals in Very High Cycle Fatigue (VHCF). The VHCF regime is associated with stress amplitudes lower than the conventional fatigue limit and numbers of cycles higher than $10^{9}$. Tests were conducted using an ultrasonic technique at loading frequency of $20 \mathrm{kHz}$. The mechanisms leading to crack initiation express via slip bands at the specimen surface and self-heating due to intrinsic dissipation. Thermal maps were used to estimate the mean dissipation and its change with number of cycles and stress amplitudes in case of pure copper polycrystals. At the same time, the surface relief changes due to plasticity were characterized using optical and scanning electronic microscopes. A good correlation was found between slip band initiation and dissipation. Dissipation and slip band amount always increased over the number of cycles. At very small stress amplitudes, no slip band appeared up to $10^{8}$ cycles but the material was found to dissipate energy. Results derived from tests performed at high loading frequency on pure cupper specimens showed a drift of dissipative regimes incompatible with concepts of fatigue limit and/or asymptotic cyclic stability. These results reveal that the material never reached a steady state. Therefore it could break at higher number of cycles.
\end{abstract}

\section{Introduction}

The very high cycle fatigue (VHCF) regime is associated with stress amplitudes lower than the conventional fatigue limit and as a result, numbers of cycles higher than $10^{9}$. The present paper deals with a fundamental research project. However, it is motivated by industrial problems. Some mechanical components, such as pistons, rotating axes, in the transportation and more generally engineering industry, have been designed previously using fatigue resistance data at lower numbers of cycles $\left(<10^{7}\right.$ cycles ; the regime of high cycle fatigue, HCF) whereas they must endure cyclic loading for a number of cycles higher than $>10^{9}$ cycles [1]. Many experiments in the literature prove that failure occurs at stress amplitudes lower than the conventional fatigue limit.

In the high cycle fatigue (HCF), cyclic strain localization in Persistent Slip Bands (PSBs) is generally observed and accepted as the critical onset of fatigue damage in ductile single-phase materials and also in many precipitation-hardened alloys [2-4]. Once formed, PSBs, accompanied by the development of extrusions and notch-like deepenings, commonly referred to intrusions, lead to cracks. For ductile single-phase materials, it is suggested that crack initiation takes place at the surface owing to the accumulation of very small but irreversible plastic deformation over very large number of cycles [3]. However, there seems to be no possibility for the formation of a pronounced PSB structure at very low stress amplitudes below the PSB threshold in the HCF regime. To better understand the relationship between loading conditions and PSBs, it is necessary to conduct additional investigations after cyclic deformation in the VHCF regime. 
In this work, we focused on pure copper which is face-centered cubic (fcc) single-phase metal. This material is a good candidate because its HCF behavior has been largely studied in literature. To perform experiments up to a very high number of cycles in a reasonable time, ultrasonic equipment at a testing frequency of $20 \mathrm{kHz}$ was used. It takes only fourteen hours to perform $10^{9}$ cycles at a testing frequency of $20 \mathrm{kHz}$, whereas it takes one hundred days at $100 \mathrm{~Hz}$. In contrast to conventional fatigue tests (low and high cycle fatigues), detecting irreversible microstructural changes using cyclic stress-strain curves analysis is no more possible, at least with conventional measurement systems, since the macroscopic behavior is quasi-elastic. To cope with this problem, we proposed to use the energy signature of the deformation mechanisms. The temperature increase of the specimen was measured during the cyclic loading. The dissipated energy was deduced from temperature field measurements by solving the heat equation accounting for exchanges losses and material conductivity. This calorimetric method has been successfully developed for conventional fatigue at testing frequencies ranging from 10-50 Hz [5-9]. In this project, it has been adapted to $20 \mathrm{kHz}$ cycling loading. From an industrial point of view, ultrasonic fatigue testing and dissipation estimates during cycling loading may appear as rapid methods to select or to class materials with regard to their fatigue properties.

\section{Material and experimental procedure}

Material and specimens. Commercial polycrystalline copper in initial form of a hot rolled plate of $14 \mathrm{~mm}$ of thickness was tested. The specimens were extracted from the center of the plate and the microstructure of material is presented in Fig.1. The mean grain size was about $30 \mu \mathrm{m}$ with a lot of annealed twins. The material is quasi-isotropic textured. The present study was conducted by using an ultrasonic fatigue machine at $20 \mathrm{kHz}$ with cylindrical and flat hourglass shaped specimens. The specimen dimensions were calculated so that the free resonant frequency of specimen in the first longitudinal mode is $20 \mathrm{kHz}$. All specimens were treated at $250^{\circ} \mathrm{C}$ for $60 \mathrm{~min}$ to relieve the residual stress (ASM specialty Hand book) without change of microstructure. Then, a procedure of mechanical and electrolytical polishing was executed till remove all hardened layers on surface specimen. As a result, before testing, the specimens were mirror polished and without residual stresses. The dynamic Young modulus in calculations was $E_{d}=130 \mathrm{GPa}$ [10]. The stress/strain amplitude along the specimen was estimated by a one-dimensional elastic calculation. It was in a good agreement with the experimental measurement obtained by the strain gages. The geometry and the stress amplitude distribution in specimens are presented in Fig.2.

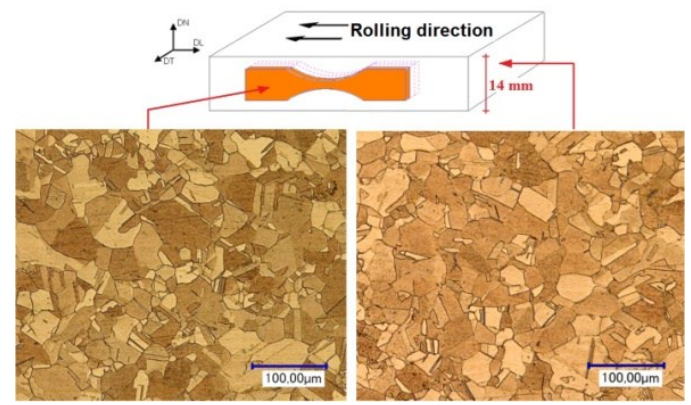

Fig.1. Microstructure of material
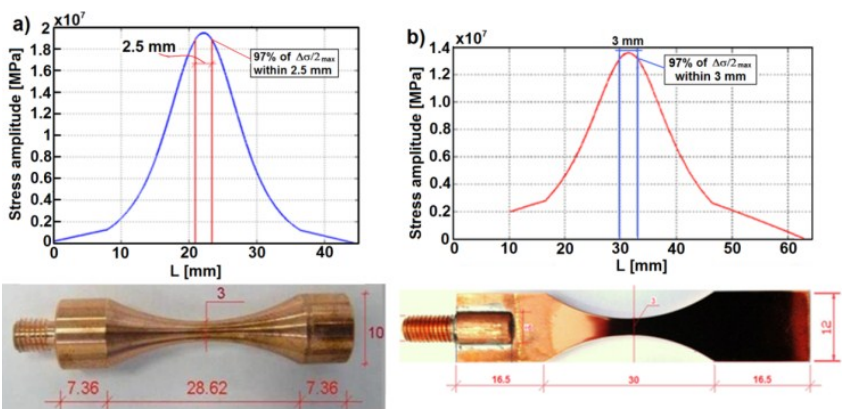

Fig.2. Ultrasonic fatigue specimens (a) cylindrical specimen (b) flat specimen

Experimental procedure. To perform experiments up to the VHCF regime, an ultrasonic equipment at a testing frequency of $20 \mathrm{kHz}$ was used. The S-N curve of the material in the VHCF regime was first established. The fatigue tests were carried-out with cylindrical specimens at various stress amplitudes with a constant stress ratio $\left(\sigma_{\max } / \sigma_{\min }\right) \mathrm{R}=-1$. In order to prevent from a heating effect at high stress amplitudes, the specimens were cooled by a cold air gun during the test till rupture. To study the formation and development of slip markings (SMs) on the specimen 
surface at very low stress amplitudes and after very high numbers of cycles, the flat specimens were used. This type of specimen is appropriate to study the microstructure and temperature and intrinsic dissipation by an infrared camera. The experiments were periodically interrupted after several specified numbers of cycles for surface observations.

\section{Calorimetric analysis}

Heat diffusion model. A numerical model was built to estimate the evolution of intrinsic dissipation fields from the temperature measurement fields along the fatigue test. A making of longitudinal distributions of heat sources within the specimen gage part was proposed. A 1D calorimetric analysis has been justified assuming in a first approximation a uniaxial tensioncompression stress state. From the heat equation:

$\rho C \dot{T}-k \Delta T=s$

where $T$ is the temperature, $\rho$ the mass density, $C$ the heat capacity, $k$ the thermal conduction coefficient and $s(x, y, z, t)$ symbolizes the volume heat source, following classical hypotheses [5], the $1 \mathrm{D}$ diffusion equation for a non-constant cross-section can be written as [6]:

$$
\rho C\left(\frac{\partial \overline{\bar{\theta}}}{\partial t}+\frac{\overline{\bar{\theta}}}{\tau_{t h}^{1 D}}\right)-k\left(\frac{\partial^{2} \overline{\bar{\theta}}}{\partial x^{2}}+\frac{\partial \overline{\bar{\theta}}}{\partial x} \frac{S^{\prime}}{S}\right)=\overline{\bar{d}}_{1}+\overline{\bar{s}}_{t h e}
$$

where the mean temperature variation over the cross-section $\overline{\bar{\theta}}(x, t)=T-T_{0}$ is identified with the surface temperature, $S^{\prime}$ being the first derivative of the cross-section $S$ while $\overline{\bar{d}}_{1}(x, t)$ and $\bar{s}_{\text {the }}(x, t)$ are the longitudinal profiles of dissipation and thermoelastic sources. Moreover the time constant $\tau_{t h}^{1 D}$ characterizes the lateral heat exchanges by conduction, convection and radiation between the specimen and the surroundings. These exchanges are assumed to be proportional to the temperature variation (linear Fourier boundary conditions). In order to estimate the left-hand side of Eq. (2), longitudinal temperature profiles were built by averaging the surface temperature over the specimen width.

Taking the loading frequency $(20 \mathrm{kHz})$ and the maximum frame rate of the IR camera $(100$ $\mathrm{Hz}$ ) into account, it must be stressed that the thermoelastic source amplitudes are, here, out of reach. Only the mean dissipation per cycle will be derived from the discrete, noisy thermal data.

Dissipation estimate. To compute $d_{1}$, the different partial derivative operators were estimated using spatio-temporal fitting. The local approximation functions were chosen as:

$\theta^{a p p}(x, t)=P_{1}(x) t+P_{2}(x)$

where $P_{1}(x)$ and $P_{2}(x)$ are second-order polynomials in $x$. These polynomials are identified over each approximation zone using a local least-squares method. The approximated thermal field is then used to compute the intrinsic dissipation, via an estimate of the time and space partial derivative operators of the heat equation (see Eq.2) [6].

\section{Results}

S-N Curve. The S-N curve of the studied pure copper obtained by ultrasonic fatigue machine at $20 \mathrm{kHz}$ is shown in Fig. 3. It displays two regimes. In the first regime, failure occured between $3.6 \times 10^{6}$ and $5 \times 10^{7}$ cycles with stress amplitude ranging from $115 \mathrm{MPa}$ to $97 \mathrm{MPa}$. The first regime is associated with the high cycle fatigue (HCF) regime. In the second regime, failure took place at numbers of cycles greater than $5 \times 10^{7}$ cycles. The stress amplitude is lower than 97 
MPa. The S-N curve slope in VHCF is more than 10 times smaller than in HCF. In other words, the number of cycles very quickly increases with decreasing the stress amplitude. At $\Delta \sigma / 2=91.2 \mathrm{MPa}$, we did not observe any failure up to $5.4 \times 10^{9}$ cycles at which the test was stopped. Our results are in very good agreement with Stanzl-Tschegg et al. [10]'s experiment results obtained on similar pure polycrystalline copper. Their results revealed a "fatigue limit at $1 \times 10^{10}$ cycles" also called "fatigue life threshold" equal to at $\Delta \sigma / 2=92.2 \mathrm{MPa}$.

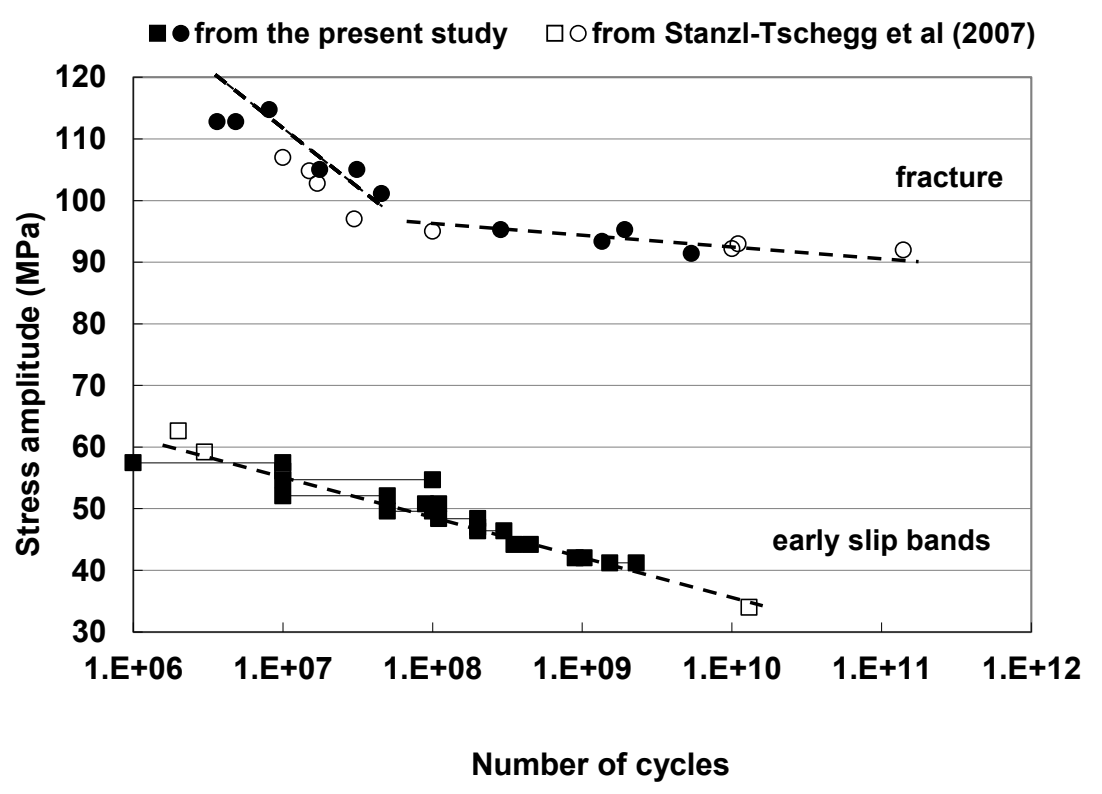

Fig.3. S-N curves to rupture and to form the early slip markings (slip bands) - Open and full circles and squares correspond to the fracture of the specimen and the appearance of the early slip bands, respectively.

Three types of persistent slip markings in fatigue. The appearance and evolution of slip markings (SM) in the VHCF regime for a wide range of stress amplitude from $38 \mathrm{MPa}$ to $115 \mathrm{MPa}$ have been studied. It was found that the lower the stress amplitude, the higher the number of cycles necessary to observe the early SMs. Besides, we distinguished 3 types of SMs: type I, type II and type III which differ from their location within the microstructure and their morphology (Fig. 3). All these SMs were shown to be Persistent Slip Markings (PSM) and are Persistent Slip Bands. The appearance of the different types of slip markings depends essentially on stress amplitude. At high stress amplitudes, i.e. higher than "fatigue limit at $1 \times 10^{10}$ cycles" at $\Delta \sigma / 2=92.2 \mathrm{MPa}$, the majority of the first slip markings is of type I. The amount of the PSM type I decreases with decreasing stress amplitudes and the PSM type II become more and more dominant. At very low stress amplitudes such as $44 \mathrm{MPa}$, type III is dominant. All kinds of PSMs (I,II and III) have been reported before [e.g. 10-11]. However, here these three types of PSMs are classified here in terms of the stress amplitude ranges at which they appear predominantly. Whatever the type of PSMs, their amount was found to increase with increasing the number of cycles. 

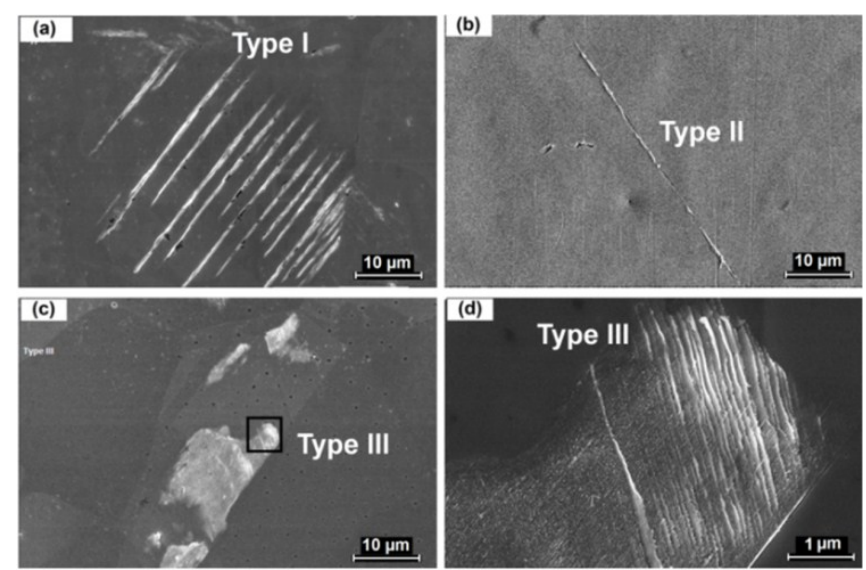

Fig.4. Three types of slip markings in VHCF (a) Type I ; (b) Type II ; (c) Type III and its zoom (d)

Intrinsic dissipation. Figure 5 shows the intrinsic dissipation distribution (divided by $\rho C$ in order to have volume energy rate $\left(\mathrm{W} / \mathrm{m}^{3}\right)$ expressed in $\left.\left({ }^{\circ} \mathrm{C} / \mathrm{s}\right)\right)$ along the specimen axis obtained for maximal stress amplitude of $72.1 \mathrm{MPa}$ after $10^{7}$ cycles. The intrinsic dissipation is concentrated in the middle of the specimen and decreases toward the ends. Hence, it is, as expected, related to the distribution of stress (Fig.2). Optical micrographs revealed that this zone display the highest quantity of slip markings (Fig. 5). This result indicates that dissipation is related to microplasticity.
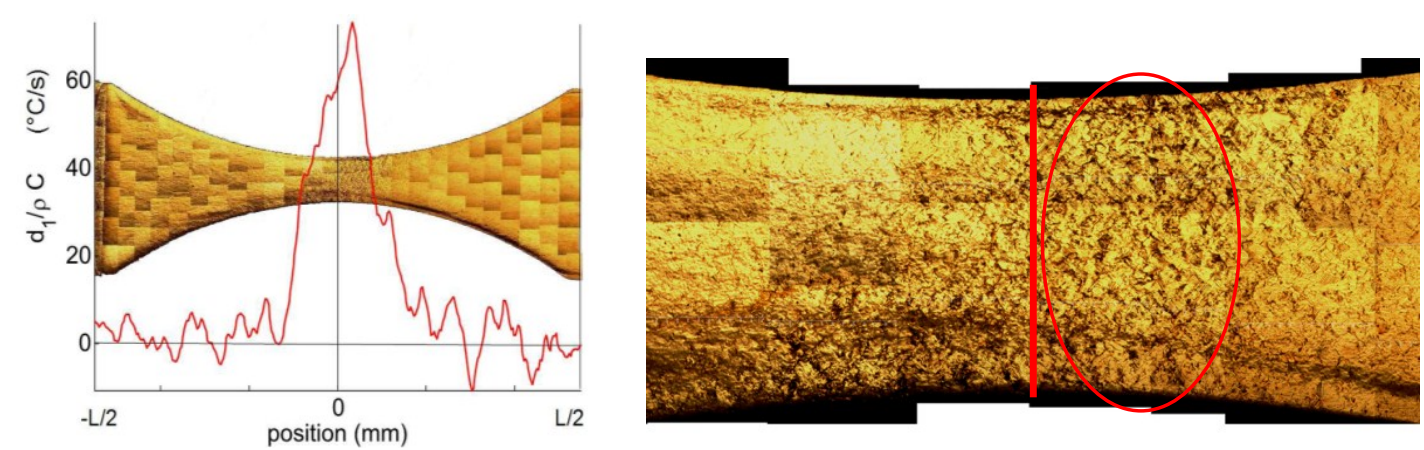

Fig. 5: Dissipation along the specimen at $\Delta \sigma / 2=72.1 \mathrm{MPa}, 10^{\prime}$ cycles and a zoom at the middle of specimen

The surface average intrinsic dissipation was estimated from thermal field measurements over fatigue tests. Fig. 6 exhibits the intrinsic dissipation $d_{1}$ at $10^{6}$ cycles for various stress amplitudes ranging from 20 to $90 \mathrm{MPa}$. Results stressed that dissipated energy exists whatever the applied load. Higher the stress range, higher the intrinsic dissipation. We can note that even below about $30 \mathrm{MPa}$, at which no slip marking appear even at number of cycles higher than $10^{10}$ and sometimes considered as an "irreversible threshold" in literature [10], a non-zero intrinsic dissipation can be detected (Fig. 6 (b)). It was also found that dissipation always increased with the number of cycles revealing continuous changes within the material. This result is consistent with the gradual increase of the PSM (slip bands) amount on the specimen surface over cycles.

\section{Conclusion}

Microstructural change of commercially pure polycrystalline copper after $20 \mathrm{kHz}$ fatigue tests in the very high cycle fatigue regime was characterized by coupling two methods: (1) observing the specimen surface relief during cycling and (2) estimating the intrinsic dissipation from temperature measurements. A good correlation was found between slip activity and dissipation. Dissipation and slip band amount always increased over the number of cycles. At very small stress amplitudes, no slip band appeared up to $10^{8}$ cycles but the material was found to 
dissipate energy. Results derived from tests performed at high loading frequency on pure cupper specimens showed a drift of dissipative regimes incompatible with concepts of fatigue limit and/or asymptotic cyclic stability. These results reveal that the material never reached a steady state. Therefore it could break at higher number of cycles.

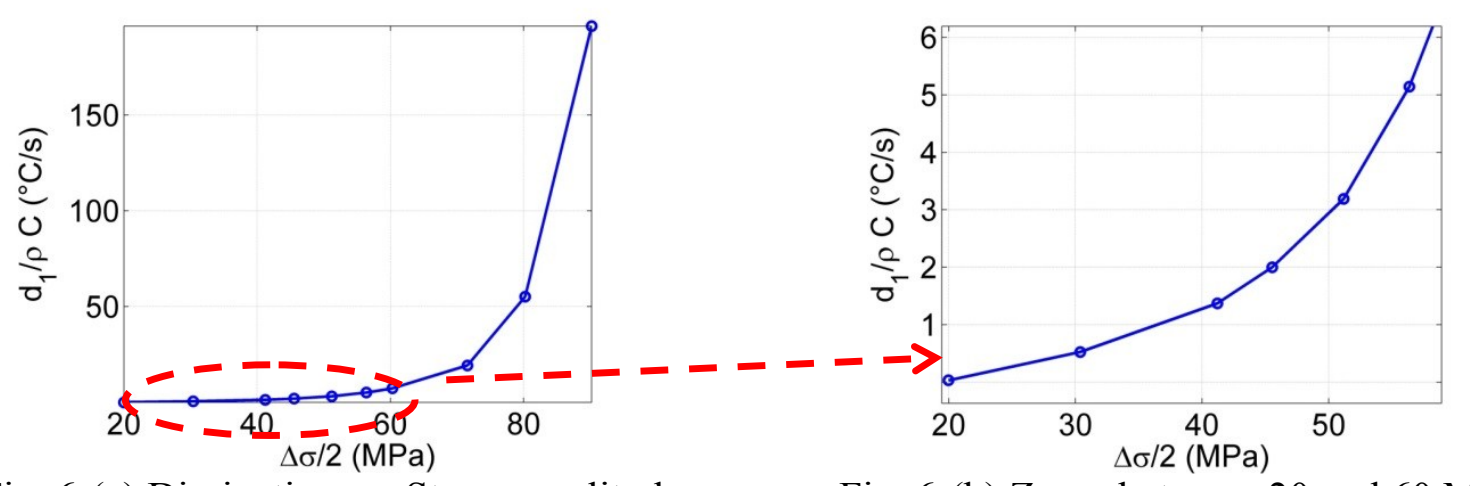

Fig. 6-(a) Dissipation vs. Stress amplitude

Fig. 6-(b) Zoom between 20 and $60 \mathrm{MPa}$

\section{Acknowledgements}

The authors are grateful for financial support from Agence Nationale de la Recherche France ANR-09-BLAN-0025-01 and to company Griset for supplying copper.

\section{References}

[1] C. Bathias, P.C. Paris, Gigacycle Fatigue in Mechanical Practice, Marcel Dekker, New York, 2005

[2] H. Mughrabi, On multi-stage fatigue life diagrams and the relevant life controlling mechanisms in ultrahigh-cycle fatigue. Fatigue Fract Engng Mater Struct, 25 (2002), 755-764.

[3] H. Mughrabi, Cyclic Slip Irreversibilities and the Evolution of Fatigue Damage, Metall Mater Trans A, 40 (2009)1257-1279.

[4] M. Risbet, X Feaugas, Some comments about fatigue crack initiation in relation to cyclic slip irreversibility, Engng Fracture Mech,, 75 (2008) 3511-3519.

[5] T. Boulanger, A. Chrysochoos, C. Mabru, A. Galtier, Calorimetric analysis of dissipative and thermoelastic effects associated with the fatigue behavior of steels Inter. J. Fatigue, 26 (2004) 221-229.

[6] V. Honorat, V., S. Moreau, J.M. Muracciole, B. Wattrisse, A. Chrysochoos, A calorimetric analysis of polymer behaviour using a pixel calibration of an IRFPA camera, Int. J. on Quantitative Infrared Thermography, 2 (2005)153-172.

[7] C. Mareau, V. Favier, B. Weber, A. Galtier, Influence of the free surface and the mean stress on the dissipation in steels under cyclic loading, Inter. J. Fatigue 31 (2009) 1407-1412.

[8] C. Doudard, S. Calloch, F. Hild, S.Roux, Identification of heat sources from infrared thermography : Determination of 'self- heating' in a dual-phase steel by using a dog bone sample, Mech. Mater., 42 (2010) 55-62.

[9] N. Connesson, F. Maquin, F. Pierron, F.Experimental energy balance during the first cycles of cyclically loaded specimens under the conventional yield stress - Exp.Mech. 51 (2011), 23-44.

[10]S. Stanzl-Tschegg, H. Mughrabi, B. Schönbauer, Life-time and cyclic slip of copper in the VHCF-regime, Int. J. Fatigue (2007) 2050-2059.

[11]S. Stanzl-Tchegg S.E., Schönbauer B., Mechanisms of strain localization, crack initiation and fracture of polycrystalline copper in the VHCF regime, Int. J. Fatigue, 32 (2010) 886-893. 